\title{
Some results for directed lattice walkers in a strip
}

\author{
Yao-ban Chan and Anthony J. Guttmann \\ Department of Mathematics and Statistics, University of Melbourne, Parkville, VIC 3010, Australia
}

Using a transfer matrix method, we present some results for directed lattice walkers in a horizontal strip of finite width. Some cases with two walkers in a small width are solved exactly, as are a couple of cases with vicious walkers in a small width; a conjecture is made for a case with three walkers. We also derive the general transfer matrix for two walkers. Lastly, we examine the dependence of the growth constant on the width and friendliness.

Keywords: vicious walkers, friendly walkers, transfer matrix, walkers in a strip

\section{Introduction}

The directed vicious walker problem considered here takes place on a $d$-dimensional regular lattice. Any number of walkers (which, at any point in time, are defined solely by their location) may start from specified points, then at regular intervals simultaneously take one step on the lattice in a random direction, chosen from an allowed set. We do not allow two walkers to meet; hence the term vicious. To simplify matters, here we take the lattice to be $\mathbb{Z} \times \mathbb{Z}$, rotated through an angle of $\frac{\pi}{4}$ clockwise and stretched by a factor of $\sqrt{2}$, and only allow the walkers to move forward in the $x$-direction. Then we set the starting points of the walkers to be $(0,2 i), i=0,1, \ldots, p$, where $p$ is the number of walkers. We shall use $p$ to denote the number of walkers throughout this paper. A typical vicious walker configuration under these conditions is shown in Figure 1.
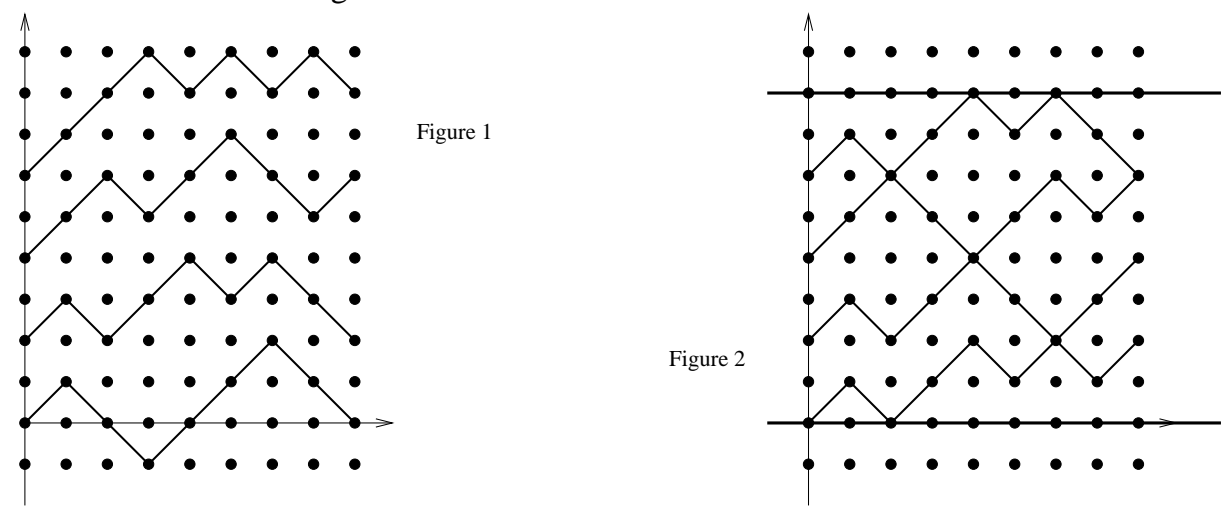

1365-8050 @ 2003 Discrete Mathematics and Theoretical Computer Science (DMTCS), Nancy, France 
The simplest question considered about this model is: how many possible configurations of walks of a fixed length are there? Failing an exact expression, we would like to find an asymptotic expression for this quantity as the length tends to infinity. This problem was considered in [GOV98], in which an exact expression for the number of configurations was found. The number of configurations grows asymptotically as $2^{m p} m^{-p^{2} / 4+p / 4}$, as $m$, the number of steps that each walker takes, becomes large.

A variant of this problem was considered in [GV02]. In that paper, the vicious constraint was relaxed, so that two walkers (but no more than two) may stay together for up to $n$ vertices. This model is called the $n$-friendly walker model, and was also studied in [KGV00], in which it was found that the number of such configurations also grows asymptotically as $2^{m p} m^{-p^{2} / 4+p / 4}$. Interestingly, this is independent of $n$, the degree of friendliness.

We can further restrict the model by not allowing the walkers to go below the horizontal line $y=0$. It was found in [KGV00] that the number of these configurations grows as $2^{m p} m^{-p^{2} / 2}$. Again this is independent of $n$.

A further restriction was added to this model and studied in [KGV03]. Under this restriction, all walkers must stay between the horizontal boundaries $y=0$ and $y=L$ for some integer $L \geq 2 p-2$. A typical configuration under such restrictions is shown in Figure 2. It was found in [KGV03] that, asymptotically, the number of $m$-step vicious walkers with these restrictions is $\frac{4^{p^{2}}}{(L+2)^{p}}\left(2^{p} \prod_{s=1}^{p} \cos \frac{s \pi}{L+2}\right)^{m}$. The case where all walkers could stay together for any number of vertices (the so-called TK $\infty$-friendly model) was also studied, and it was found that the growth constant changed, as the number of such configurations was asymptotically $\frac{4^{p^{2}}}{(L+2 p)^{p}}\left(2^{p} \prod_{s=1}^{p} \cos \frac{s \pi}{L+2 p}\right)^{m}$. This differs qualitatively from the half-plane model (bounded on the $x$-axis only) in that the growth constant is clearly $n$-dependent, although the nature of that dependence was not studied in [KGV03]. This dependence is not really surprising, as the model of walkers in a strip of finite width is essentially a one-dimensional model, whereas the half-plane model is a two-dimensional model, so fundamental differences are to be expected.

In this paper, we give some results for the model of walkers in a strip of finite width. Using the transfer matrix method given in Section 3, we were able to generate the first few terms (up to at most the 40th term) of each series for $p=2$. For higher $p$, we used a method of recurrences, devised by Gessel ([Ges]). The transfer matrix for walkers in a finite strip must be finite, so it follows that the generating functions are rational (from Theorem 4.7.2 of [Sta97]). Thus we were able to use Padé approximants to seek the exact generating function.

The Padé approximant seeks to approximate the generating function of a series, given the first few terms, by a rational function $\frac{P(x)}{Q(x)}$. If $P(x)$ is a polynomial of degree $M$, and $Q(x)$ is a polynomial of degree $N$, this is known as the $[M, N]$ Padé approximant. Without loss of generality, we can take $Q(0)=1$; thus, given a number of terms in the series, we can calculate that number plus one coefficients of $P$ and $Q$. For example, if we wished to calculate the $[N, N]$ approximant, we would require $2 N+1$ terms. In practice, we found the $[N-1, N]$ approximant usually appropriate. Indeed, in many cases we could generate sufficient terms to enable us to conjecture the exact (rational) generating function. We could then find the growth constant by evaluating the reciprocal of the smallest positive real zero of $Q(x)$.

From the generating functions, we were able to conclude that the growth constant changes monotonically with $n$. 


\section{One walker}

If there is only one walker, the model is considerably simplified, because the issue of friendliness does not arise. The generating function for this case is well known (for example, in [Kra01]). We state it again here for comparison with later results. From now on, we will assume that the range of a summation is from $-\infty$ to $\infty$ unless specified otherwise.

Theorem 2.1. In a strip of width L, the (isotropic) generating function for one walker that returns to the $x$-axis is

$$
g_{L}(x)=\frac{h_{L}(x)}{h_{L+1}(x)}
$$

and the generating function for one walker with an arbitrary end-point is

$$
f_{L}(x)=\frac{1}{h_{L+1}(x)} \sum_{i=0}^{L} x^{i} h_{L-i}(x)
$$

where

$$
h_{L}(x)=\sum_{i}(-1)^{i}\left(\begin{array}{c}
L-i \\
i
\end{array}\right) x^{2 i}
$$

is a polynomial of degree $2\left\lfloor\frac{L}{2}\right\rfloor$. If we define $\lambda_{ \pm}=\frac{1 \pm \sqrt{1-4 x^{2}}}{2}$, then this becomes

$$
g_{L}(x)=\frac{\lambda_{+}^{L+1}-\lambda_{-}^{L+1}}{\lambda_{+}^{L+2}-\lambda_{-}^{L+2}}
$$

and

$$
f_{L}(x)=\frac{1}{\lambda_{+}^{L+2}-\lambda_{-}^{L+2}}\left(\frac{\lambda_{+}^{L+1}-x^{L+1}}{1-x / \lambda_{+}}-\frac{\lambda_{-}^{L+1}-x^{L+1}}{1-x / \lambda_{-}}\right) .
$$

\section{A transfer matrix algorithm}

If we know the transfer matrix of a system, we can easily generate many series terms by repeated matrix multiplication, or we can apply a well-known result, Theorem 4.7.2 from [Sta97], which we state here as it will be useful later on.

Theorem 3.1. If A is the transfer matrix of a system, then the generating function of paths from state $i$ to state $j$ is given by

$$
\frac{(-1)^{i+j} \operatorname{det}(I-x A ; j, i)}{\operatorname{det}(I-x A)}
$$

where $\operatorname{det}(I-x A ; j, i)$ is the minor of $I-x A$ obtained by deleting the jth row and ith column and taking the determinant of the resulting matrix.

So to generate the first few terms of each series, we used the following lemma: 
Lemma 3.2. For two n-friendly walkers in a strip of width $L$, the transfer matrix has the block structure

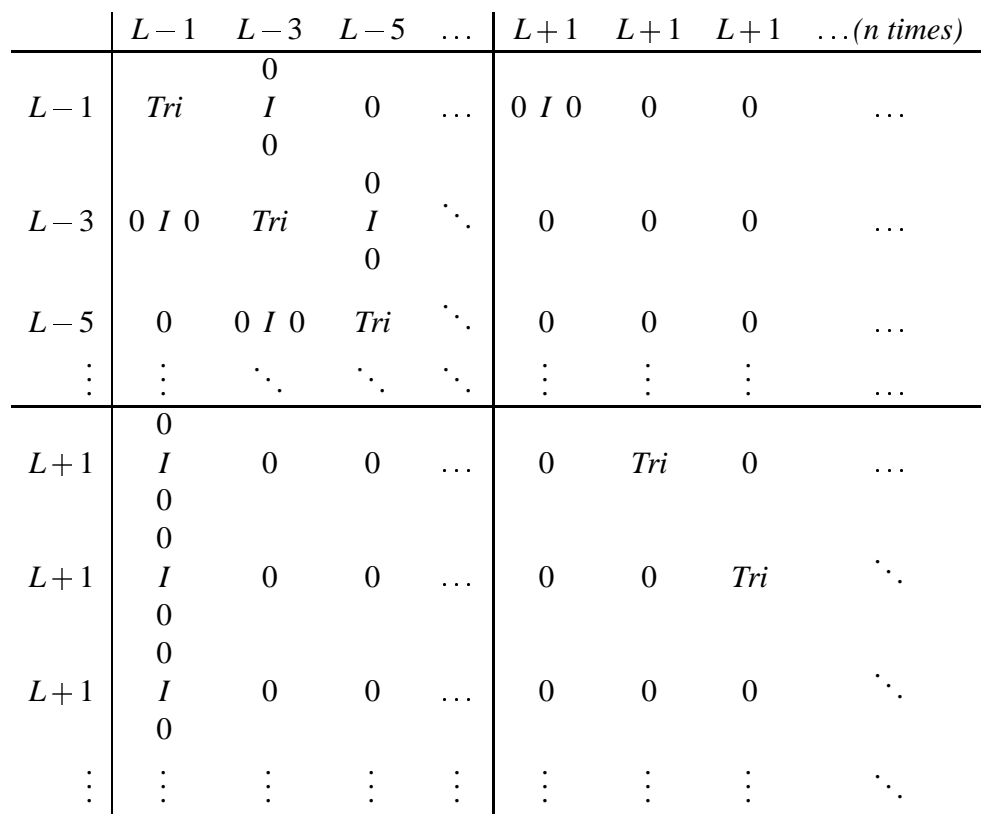

where Tri means the tri-diagonal matrix with O's on the diagonal and I's on the off-diagonals, I is the identity matrix (the dimension should be clear), and 0 is either a single row of 0 's, a single column of 0 's, or a matrix of zeros (again it should be clear which is meant). The first row and column give the widths of each block.

Proof. An important fact used implicitly in this proof is that the walkers must be an even number of units apart from each other. Also, the transfer matrix for two walkers which remain a constant distance from each other (i.e. they take the same steps, but at a fixed distance from each other) is the tri-diagonal matrix with 0's on the diagonal and 1's on the off-diagonals.

The rows and columns of the transfer matrix are labeled by the state of the system at any one time. So we set the states to be the heights of the walkers at that time. For example, a state of $(1,3)$ denotes that the first walker is at height 1 and the second walker is at height 3 .

First consider the vicious case. The transfer matrix for this case is the top left section of the above matrix. The first $L-1$ states represent the states where the walkers are two units apart - $(0,2),(1,3)$, and so on in that order. The second $L-3$ states represent the states where the walkers are four units apart - $(0,4),(1,5)$, and so on, again in that order. This continues in a similar manner, with the last entries representing walkers that are as far apart as possible. By noting that each step can only change the distance apart by $-2,0$, or 2 , it can be seen that the transfer matrix is the top left section of the above matrix.

Now consider the case of arbitrary $n$. We have the same states as in the vicious case as well as states where the walkers are together, for example $(1,1)$. However, to keep track of how long the walkers have been together, we attach a subscript to each of the latter states. This subscript gives the number of vertices that the walkers have been together prior to (and including) this vertex. Then we make the first set of $L+1$ vertices represent the states $(0,0)_{1},(1,1)_{1}$, and so on, while the second set represents 
$(0,0)_{2},(1,1)_{2}$, and so on, and this continues until we reach $(L, L)_{n}$. Observe that walkers separated by two units can step to unseparated walkers with subscript 1 only. On the other hand, unseparated walkers with arbitrary subscript can step to walkers separated by two units, but no more. Also, if walkers that are together remain together, their subscript must increase by exactly 1 . Combining these observations with the transfer matrix for vicious walkers, it can be seen that the general transfer matrix is as stated above.

By taking powers of this transfer matrix and summing the entries in the first row, we were able to enumerate the walker series many times faster than by a direct enumeration algorithm.

\section{Proofs and Conjectures}

Unsurprisingly, we were unable to find a general result for the generating function for more than one walker. However, we were able to prove formulae for two walkers in strips of width 3 and 4 .

Theorem 4.1. The generating function for two $n$-friendly walkers in a strip of width 3 is

$$
\frac{\sum_{i=0}^{n} F_{i} x^{i}}{1-x-\sum_{i=0}^{n-1} F_{i} x^{i+2}}=\frac{1-F_{n+1} x^{n+1}-F_{n} x^{n+2}}{1-2 x-x^{2}+x^{3}+F_{n} x^{n+2}+F_{n-1} x^{n+3}}
$$

where $F_{n}$ is the nth Fibonacci number $\left(F_{0}=F_{1}=1, F_{n}=F_{n-1}+F_{n-2}(n \geq 2)\right)$. This extends to $\infty$-friendly walkers which have generating function

$$
\frac{1 /\left(1-x-x^{2}\right)}{1-x-x^{2} /\left(1-x-x^{2}\right)}=\frac{1}{1-2 x-x^{2}+x^{3}}
$$

Proof. Firstly we consider the generating function of configurations where the walkers do not end at the same height. We can divide such walks at times when the walkers are 2 units apart (in other words, when they are at the states $(0,2)$ or $(1,3))$. At any such point, the walkers must either step in the same direction (which has generating function $x$ ) or come together for up to $n$ vertices, and then separate. So we need merely find the generating function for the second case. Note that since $(0,2)$ and $(1,3)$ are reflections of each other in the line $y=\frac{3}{2}$, we can identify both states as one.

We can see that the second case is composed of an initial step (where the walkers come together), a single walk starting from height 1 , ending at height 1 or 2 , and taking no more than $n-1$ steps (n vertices), and a final step (where the walkers separate). From Theorem 3.1 and the one-walker transfer matrix (which, in the notation of Lemma 3.2, is a $4 \times 4$ Tri matrix), if we remove the length restriction on the middle walk, its generating function is $\frac{1}{1-x-x^{2}}$; therefore the generating function for the second case is $x^{2} \sum_{i=0}^{n-1} F_{i} x^{i}$. Thus the entire configuration must consist of a sequence of walks with generating function $x+\sum_{i=0}^{n-1} F_{i} x^{i+2}$, and therefore its generating function is $\frac{1}{1-x-\sum_{i=0}^{n-1} F_{i} x^{i+2}}$.

Now we consider the possibility that the two walkers may end together. If we divide the configuration at the last point that the two walkers are separate, it can be seen that the generating function is of the form $\frac{1}{1-x-\sum_{i=0}^{n-1} F_{i} x^{i+2}}(1+x g(x))$, where $g(x)$ is the generating function for one walker starting at 1 and taking less than $n$ steps. Again from Theorem 3.1, the generating function for a walker starting from height 1 is $\frac{1+x}{1-x-x^{2}}=\sum_{i>0} F_{i+1} x^{i}$, so $g(x)=\sum_{i=1}^{n} F_{i} x^{i-1}$. Putting it all together, the generating function for two 
$n$-friendly walkers in a strip of width 3 is $\frac{\sum_{i=0}^{n} F_{i} x^{i}}{1-x-\sum_{i=0}^{n-1} F_{i} x^{i+2}}$. Multiplying both numerator and denominator by $1-x-x^{2}$ gives the second form of the Theorem.

Theorem 4.2. The generating function for two $n$-friendly walkers in a strip of width 4 is

$$
\begin{aligned}
& \frac{\sum_{i=0}^{n} a_{i} x^{i}-2\left(3^{\frac{n-1}{2}} x^{n+2}+\sum_{i=\frac{n+3}{2}}^{n} 3^{i-2} x^{2 i+1}\right)}{\sum_{i=0}^{\frac{n+1}{2}} b_{i} x^{2 i}+2\left(3^{\frac{n-1}{2}} x^{n+3}+\sum_{i+\frac{n+3}{2}}^{n} 3^{i-2} x^{2 i+2}\right)}=\frac{1+2 x-x^{2}-2 x^{3}+3^{k} x^{n+1}\left(-2-6 x+4 x^{3}\right)+2\left(3^{2 k}\right) x^{2 n+3}}{1-8 x^{2}+8 x^{4}+3^{k} x^{n+3}\left(9-4 x^{2}\right)-2\left(3^{2 k}\right) x^{2 n+4}} \\
& \text { if } n=2 k+1 \text { is odd, and } \\
& \frac{\sum_{i=0}^{n} a_{i} x^{i}+3^{\frac{n-2}{2}} x^{n+1}+2\left(-3^{\frac{n-2}{2}} x^{n+2}+\sum_{i=\frac{n+2}{2}}^{n} 3^{i-2} x^{2 i+1}\right)}{\sum_{i=0}^{\frac{n}{2}} b_{i} x^{2 i}-2\left(3^{\frac{n-2}{2}} x^{n+2}+\sum_{i=\frac{n+2}{2}}^{n} 3^{i-2} x^{2 i+2}\right)}=\frac{1+2 x-x^{2}-2 x^{3}+3^{k-1} x^{n+1}\left(-3-8 x-x^{2}+6 x^{3}\right)-2\left(3^{2 k-1}\right) x^{2 n+3}}{1-8 x^{2}+8 x^{4}+3^{k-1} x^{n+2}\left(5+4 x^{2}\right)+2\left(3^{2 k-1}\right) x^{2 n+4}}
\end{aligned}
$$

if $n=2 k$ is even, where $a_{i}$ are coefficients defined by $a_{0}=1, a_{1}=2, a_{2 i}=2(3)^{i-1}(i \geq 1)$, and $a_{2 i+1}=$ $4(3)^{i-1}(i \geq 1)$, and $b_{i}$ are coefficients defined by $b_{0}=1, b_{1}=-5$, and $b_{i}=-7(3)^{i-2}(i \geq 2)$. This also extends to the $\infty$-friendly case, which has generating function

$$
\frac{1+2 x+2 x^{2}(1+2 x) /\left(1-3 x^{2}\right)}{1-5 x^{2}-7 x^{4} /\left(1-3 x^{2}\right)}=\frac{1+2 x-x^{2}-2 x^{3}}{1-8 x^{2}+8 x^{4}} .
$$

Proof. In this model, there are 4 possible states where the walkers are separate - $(0,2),(1,3),(2,4)$, and $(0,4)$. We can divide the walks according to these states, which then allows us to construct a modified transfer matrix using these states as the only states. The entries of this matrix are the generating functions of the possible walks from one state to another, without attaining any of the states in between. It is convenient, however, to identify the states $(0,2)$ and $(2,4)$ with each other as they are reflections of each other in the line $y=2$. So we will take the states in the order $(0,2) /(2,4),(1,3),(0,4)$.

To find each entry in the matrix, there are two possibilities: if the start state can move to the end state with one move, there is a factor of $x$, and if the walkers are 2 units apart in both start and end states, they may come together for up to $n$ vertices and then separate, which results in a factor of $x^{2}$ multiplied by the generating function for one walker in a strip of width 4 starting and ending at the appropriate points, and not exceeding $n-1$ steps. For example, the entry $(0,2) /(2,4) \rightarrow(1,3)$ of the matrix would be $x+x^{2} g(x)$, where $g(x)$ is the generating function of one walker in a strip of width 4 starting at height 1 , ending at height 2 , and not taking more than $n-1$ steps. Such a function can be derived from the one-walker transfer matrix and Theorem 3.1. We find that the modified transfer matrix is

$$
\left(\begin{array}{ccc}
\sum_{i=0}^{\left\lfloor\frac{n-1}{2}\right\rfloor} 3^{i} x^{2 i+2} & x+\sum_{i=0}^{\left\lfloor\frac{n-2}{2}\right\rfloor} 3^{i} x^{2 i+3} & 0 \\
2 x+2 \sum_{i=0}^{\left\lfloor\frac{n-2}{2}\right\rfloor} 3^{i} x^{2 i+3} & \frac{1}{3} x^{2}+\frac{2}{3} \sum_{i=0}^{\left\lfloor\frac{n-1}{2}\right\rfloor} 3^{i} x^{2 i+2} & x \\
0 & x & 0
\end{array}\right)=\left(\begin{array}{ccc}
x^{2} \frac{1-\left(3 x^{2}\right)^{\left\lfloor\frac{n+1}{2}\right\rfloor}}{1-3 x^{2}} & x+x^{3} \frac{1-\left(3 x^{2}\right)^{\left.\frac{n}{2}\right\rfloor}}{1-3 x^{2}} & 0 \\
2 x+2 x^{3} \frac{1-\left(3 x^{2}\right)^{\left\lfloor\frac{n}{2}\right\rfloor}}{1-3 x^{2}} & \frac{1}{3} x^{2}+\frac{2}{3} x^{2} \frac{1-\left(3 x^{2}\right\rfloor^{\left\lfloor\frac{n+1}{2}\right\rfloor}}{1-3 x^{2}} & x \\
0 & 0
\end{array}\right)
$$

Now we can apply Theorem 3.1 again, but replace $x A$ with our modified transfer matrix, to find the generating function of $n$-friendly walks to each of the states. To find the total generating function, we 
need merely multiply each of these by $1+x g(x)$, where $g(x)$ is the generating function for one walker, starting at an appropriate height, and not traveling more than $n-1$ steps, and then add the results together. We will assume that $n=2 k$ is even; the proof is similar for $n$ odd. The appropriate functions are:

$$
\begin{array}{r|ccc}
\text { State } & (0,2) /(2,4) & (1,3) & (0,4) \\
\text { gf to state } & \frac{1-5 x^{2}+4 x^{4}+2\left(3^{k-1}\right) x^{n+2}}{d(x)} & \frac{x-2 x^{3}-3^{k} x^{n+3}}{d(x)} & \frac{x^{2}-2 x^{4}-3^{k} x^{n+4}}{d(x)} \\
\mathrm{g}(\mathrm{x}) & \frac{1+2 x-(1+2 x)\left(3 x^{2}\right)^{k}}{1-3 x^{2}} & \frac{3+6 x+3 x^{2}-(4+6 x)\left(3 x^{2}\right)^{k}}{3\left(1-3 x^{2}\right)} & 0
\end{array}
$$

where $d(x)$ denotes the denominator of the second form of the Theorem. Multiplying these functions and adding results in that form. Dividing both numerator and denominator by $1-3 x^{2}$ results in the first form.

Interestingly, there appears to be a pattern similar in nature for three $n$-friendly walkers in a strip of width 4. This currently remains a conjecture.

Conjecture 4.3. The generating function for three $n$-friendly walkers in a strip of width 4 is

$$
\frac{\sum_{i=0}^{n} a_{i} x^{i}-5 \sum_{i=\frac{n+2}{2}}^{n} 3^{i-2} x^{2 i}}{\sum_{i=0}^{n} b_{i} x^{2 i}+\sum_{i=\frac{n+2}{2}}^{n} 3^{i-2} x^{2 i}}=\frac{1+2 x+2 x^{2}+3^{k-1} x^{n+1}(-6-20 x)+5\left(3^{2 k-1}\right) x^{2 n+2}}{1-8 x^{2}+16\left(3^{k-1}\right) x^{n+2}-3^{2 k-1} x^{2 n+2}}
$$

if $n=2 k$ is even, and

$$
\frac{\sum_{i=0}^{n} a_{i} x^{i}+3^{\frac{n-1}{2}} x^{n+1}+5 \sum_{i=\frac{n+3}{2}}^{n} 3^{i-2} x^{2 i}}{\sum_{i=0}^{\frac{n-1}{2}} b_{i} x^{2 i}-3^{\frac{n+1}{2}} x^{n+1}-\sum_{i=\frac{n+3}{2}}^{n} 3^{i-2} x^{2 i}}=\frac{1+2 x+2 x^{2}+3^{k} x^{n+1}\left(-4-6 x+2 x^{2}\right)-5\left(3^{2 k}\right) x^{2 n+2}}{1-8 x^{2}+3^{k} x^{n+1}\left(2+8 x^{2}\right)+3^{2 k} x^{2 n+2}}
$$

if $n=2 k+1$ is odd, where $a_{i}$ are coefficients defined by $a_{0}=1, a_{2 i-1}=2\left(3^{i-1}\right)(i \geq 1)$, and $a_{2 i}=$ $5\left(3^{i-1}\right)(i \geq 1)$, and $b_{i}$ are coefficients defined by $b_{0}=1, b_{i}=-5\left(3^{i-1}\right)(i \geq 1)$. Again, this extends to the $\infty$-friendly case, which has generating function

$$
\frac{1+\left(2 x+5 x^{2}\right) /\left(1-3 x^{2}\right)}{1-5 x^{2} /\left(1-3 x^{2}\right)}=\frac{1+2 x+2 x^{2}}{1-8 x^{2}} .
$$

There also appears to be a similar pattern for higher widths - both numerator and denominator have 'fixed' coefficients, which are coefficients of the lower powers of $x$ that do not change as $n$ increases. Every time $n$ increases, it appears that both numerator and denominator acquire one more 'fixed' coefficient. If we can extend these 'fixed' coefficients into an infinite series, we derive the $\infty$-friendly generating function. However, for finite $n$ and $L>3$, 'unfixed' coefficients also appear.

Finally, for relatively small widths, some results can be proved for vicious walks with any number of walkers:

Theorem 4.4. The generating function for $p$ vicious walkers in a strip of width $2 p-1$ is

$$
\frac{1}{1-x}
$$


The generating function for $p$ vicious walkers in a strip of width $2 p$ is

$$
\frac{1+x}{1-(p+1) x^{2}} \text {. }
$$

Proof. The first result is obvious, as there is only one possible configuration of walks at any stage.

For the second result, at positions with odd $x$-coordinate there is only one possible state that the walkers can be in. At positions with even $x$-co-ordinate, there are $p+1$ possible states. This can be seen by observing that there are $p+1$ points in the strip with even height, so there must be one 'empty' space which can be placed in any of $p+1$ places. All of these states can be reached from and can go to the only possible state with odd $x$-coordinate. Thus the number of configurations is a sequence $1,1, p+1, p+$ $1,(p+1)^{2},(p+1)^{2}, \ldots$ The generating function now follows.

Theorem 4.5. The generating function for $p$ vicious walkers in a strip of width $2 p+1$ is

$$
f_{p}(x)=\frac{h_{p-1}(x)}{h_{p+1}(x)}
$$

where

$$
h_{p}(x)=\sum_{i}(-1)^{\left\lfloor\frac{i+1}{2}\right\rfloor}\left(\begin{array}{c}
\left\lfloor\frac{p+i}{2}\right\rfloor \\
i
\end{array}\right) x^{i}
$$

is a polynomial of degree $p$. Alternately if we define $\lambda_{ \pm}=\frac{2-x^{2} \pm x \sqrt{x^{2}-4}}{2}$, then

$$
h_{p}(x)=\left(\frac{1}{2}-\frac{2 x+x^{2}}{2 x \sqrt{x^{2}-4}}\right) \lambda_{+}^{k}+\left(\frac{1}{2}+\frac{2 x+x^{2}}{2 x \sqrt{x^{2}-4}}\right) \lambda_{-}^{k}
$$

if $p=2 k$ is even, and

$$
h_{p}(x)=\left(\frac{1-x}{2}-\frac{2 x+x^{2}-x^{3}}{2 x \sqrt{x^{2}-4}}\right) \lambda_{+}^{k}+\left(\frac{1-x}{2}+\frac{2 x+x^{2}-x^{3}}{2 x \sqrt{x^{2}-4}}\right) \lambda_{-}^{k}
$$

if $p=2 k+1$ is odd.

Proof. A number of properties of the conjectured generating function are instrumental in proving this theorem. For convenience we state these as a separate lemma.

Lemma 4.6. If $f_{p}(x)$ and $h_{p}(x)$ are as stated in Theorem 4.5, then
1. $h_{p}(x)=h_{p-2}(x)-x h_{p-1}(-x)$
3. $h_{p}(x)=\left(2-x^{2}\right) h_{p-2}(x)-h_{p-4}(x)$
2. $h_{p}(x)+h_{p-2}(x)=\left(2+(-1)^{p} x\right) h_{p-1}(x)$
5. $f_{p}(x)=\frac{1+f_{p-1}(x)}{3-x^{2}-f_{p-1}(x)}$
4. $f_{p}(x)=\frac{1}{2-x^{2}-f_{p-2}(x)}$
6. $f_{p}(-x)=\left(\frac{f_{p-1}(x)-1}{f_{p+1}(x)-1}\right) f_{p+1}(x)$

These properties can be proved relatively easily by algebraic manipulation; the latter three properties follow from the earlier three. To prove the Theorem itself, we will find a recurrence that the actual generating function satisfies, and prove that the conjectured generating function also satisfies it.

Note that at any particular time, there are exactly $p+1$ possible positions for the walkers to be in. This can be seen by observing that the walkers must have either all odd or all even $y$-coordinates; in either case, 
there are $p+1$ such $y$-coordinates in a strip of width $2 p+1$, which means that there are $p+1$ choices for the only unoccupied $y$-coordinate (or 'hole'), and thus $p+1$ choices for the entire set of walkers. Note in particular that if the first (lowest) walker has $y$-coordinate 2 or 3, the no-crossing constraint ensures that the lone 'hole' is below the first walker, and thus there is exactly 1 possible arrangement for all the walkers, namely $(2,4, \ldots, 2 p)$ if the first walker is at height 2 or $(3,5, \ldots, 2 p+1)$ otherwise, where we use the same notation as Lemma 3.2 to denote the positions of the walkers.

So let the actual generating function that we seek be $g_{p}(x)$. We construct a recurrence by considering the generating function of walks that start from $(2,4, \ldots, 2 p)$. This position is the only position possible that can be achieved in one step from $(3,5, \ldots, 2 p+1)$. But the generating function of walks from that position is equal to $g_{p}(x)$, since the position is merely a reflection in $y=p+\frac{1}{2}$ of the required starting position $(0,2, \ldots, 2 p-2)$. So the generating function of walks starting from $(2,4, \ldots, 2 p)$ is $g_{p}(x)$ with the first step taken off, i.e. $\tilde{g}_{p}(x)=\frac{g_{p}(x)-1}{x}$.

Now we divide such walks into separate parts to generate the recurrence. More specifically, we separate the walks at each point where the first walker returns to a height of 2 . There are 2 possible paths that the first walker can take to return to 2, which are shown in Figure 3.

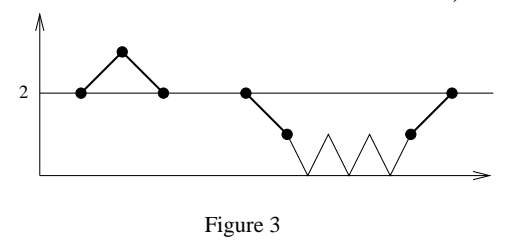

Because the highest $p-1$ walkers have only one position to go to when the first walker is at height 2 or 3 , the first possibility has generating function $x^{2}$.

The second possibility is more complicated. We can say that since the first walker starts at 2 , the starting position must be $(2,4, \ldots, 2 p)$. In particular, the highest $p-1$ walkers start from $(4,6, \ldots, 2 p)$. Now we can consider the highest $p-1$ walkers, from the starting point to one step before the first walker returns to 2 , as walkers in a strip of width $2(p-1)+1=2 p-1$, namely the strip from $y=2$ to $y=2 p+1$, which start from $(4,6, \ldots, p-1)$. This is valid because the first walker only goes above 1 at the start, which does not interfere with the starting position, and at all other points it is either at 0 or 1 , and if it is at height 0 then it is impossible to have a walker at height 1 . But this is the same as $p-1$ vicious walkers in a strip of width $2 p-1$ that start from $(2,4, \ldots, 2 p-2)$, which has a generating function of $\tilde{g}_{p-1}(x)$ from above.

However, there are restrictions on the length of such configurations. In particular, the walks must be of length at least one, as the first walker takes at least one step. This step is still counted in the generating function, so we need merely subtract 1 from the generating function. Also the walks must be of odd length, as they must end when the first walker is at 1 and not 0 . To achieve this we subtract $\tilde{g}_{p-1}(-x)-1$ and divide by 2 . Lastly we must multiply by a factor of $x$ for the (previously) uncounted last step. We can do this because for all possible configurations with the first walker at height 1 , there is exactly one way to go to the single configuration with the first walker at height 2 . So the generating function for the second possibility is

$$
\frac{1}{2} x\left(\tilde{g}_{p-1}(x)-1-\tilde{g}_{p-1}(-x)+1\right)=\frac{1}{2}\left(g_{p-1}(x)+g_{p-1}(-x)\right)-1 .
$$

Having considered the possible ways in which the first walker can return to 2 , we must consider the possible ways in which it can end, having started at 2 and not returned there. There are four possible configurations, as shown in Figure 4. 


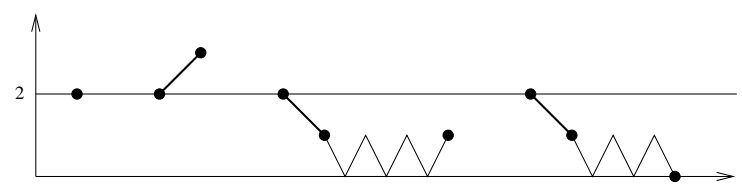

Figure 4

The first 2 possibilities have obvious generating functions of 1 and $x$ respectively, as again there is only one possibility for the remaining walkers if the first walker is at 2 or 3 . The other two can be counted as one possibility, and again we can use the same argument with the remaining walkers. However, this time there is no extra factor of $x$, and the walkers need not be of odd length, but they still have to have at least length 1 . Thus the generating function for these possibilities is $\tilde{g}_{p-1}(x)-1$.

Putting all this together, we need merely observe that any configuration consists of an arbitrary number of segments where the first walker returns to 2 , followed by exactly one end-segment, to derive the recurrence

$$
\frac{g_{p}(x)-1}{x}=\frac{1}{1-\left(x^{2}+\frac{1}{2}\left(g_{p-1}(x)+g_{p-1}(-x)\right)-1\right)}\left(1+x+\frac{g_{p-1}(x)-1}{x}-1\right)
$$

and therefore

$$
g_{p}(x)=\frac{1+\frac{1}{2}\left(g_{p-1}(x)-g_{p-1}(-x)\right)}{2-x^{2}-\frac{1}{2}\left(g_{p-1}(x)+g_{p-1}(-x)\right)} .
$$

Since $g_{1}(x)=f_{1}(x)=\frac{1}{1-x-x^{2}}$, all that remains is to show that $f_{p}(x)$ satisfies the above recurrence. This is equivalent to showing that

$$
\left(4-2 x^{2}\right) f_{p}(x)-f_{p}(x) f_{p-1}(x)-f_{p}(x) f_{p-1}(-x)-f_{p-1}(x)+f_{p-1}(-x)-2=0 .
$$

This can be shown by straightforward algebraic manipulation upon applying Lemma 4.6, parts 4, 5, and 6.

The alternate form of $h_{p}(x)$ can be derived from calculating the function $\sum_{p} h_{p}(x) y^{p}=\frac{1+(1-x) y-(1+x) y^{2}-y^{3}}{1-\left(2-x^{2}\right) y^{2}+y^{4}}$, which is easily derived from Lemma 4.6, part 3. Expanding in partial fractions gives the alternate form.

Corollary 4.7. If $h_{p}(x)$ is as defined in Theorem 4.5, then

$$
h_{p+1}\left(\frac{1}{2} \sec \frac{(p+1) \pi}{2 p+3}\right)=0 .
$$

Proof. This follows from the fact that $2^{p} \prod_{s=1}^{p} \cos \frac{s \pi}{2 p+3}=\frac{1}{2} \sec \frac{(p+1) \pi}{2 p+3}$ is the growth constant for $p$ vicious walkers in a strip of width $2 p+1$, and $h_{p+1}(x)$ is the denominator of the generating function for those objects.

It is worth noting that the growth constant for $p$ vicious walkers in a strip of width $L \geq 2 p-2$ (which is $2^{p} \prod_{s=1}^{p} \cos \frac{s \pi}{L+2}$ ) has the somewhat simpler form

$$
2^{p} \prod_{s=1}^{p} \cos \frac{s \pi}{2 p+2 k}=2^{1-k} \sqrt{p+k} \prod_{i=1}^{k-1} \sec \frac{(p+i) \pi}{2 p+2 k}
$$


if $L=2 p+2 k-2$ is even and

$$
2^{p} \prod_{s=1}^{p} \cos \frac{s \pi}{2 p+2 k+1}=2^{-k} \prod_{i=1}^{k} \sec \frac{(p+i) \pi}{2 p+2 k+1}
$$

if $L=2 p+2 k-1$ is odd.

\section{Growth constants for $p=2$}

From Corollary 7 of [KGV03], the growth constant for two vicious walkers in a strip of width $L$, which we will denote as $\mu_{2,0}(L)$, is asymptotically $4 \cos \frac{\pi}{L+2} \cos \frac{2 \pi}{L+2}$. From Corollary 8 of the same paper, the growth constant for two TK $\infty$-friendly walkers in a strip of width $L$ is asymptotically $\mu_{2, \infty}(L) \sim 4 \cos \frac{\pi}{L+4} \cos \frac{2 \pi}{L+4}$. Our calculations show that the growth constant for two $n$-friendly walkers, $\mu_{2, n}(L)$, interpolates monotonically between these two limits. Therefore it seems possible that for two $n$-friendly walkers, the growth constant will asymptotically be $\mu_{2, n}(L) \sim 4 \cos \frac{\pi}{L+\lambda_{2}(n)} \cos \frac{2 \pi}{L+\lambda_{2}(n)}$, where $\lambda_{2}(n)$ is 2 for $n=0$ and 4 for $n=\infty$. Analysis of the generated sequences for $L$ up to 14 seems to support this conclusion, and indicates that $\lambda_{2}(n)$ increases monotonically with $n$. The values of $\lambda_{2}(n)$ were estimated to be:

\begin{tabular}{l|cccccc}
$n$ & 0 & 1 & 2 & 3 & 4 & $\infty$ \\
\hline$\lambda_{2}(n)$ & 2 & 2.67 & 3.2 & 3.54 & 3.7 & 4
\end{tabular}

From analyses of cases with $p>2$, we can say that $\lambda_{p}(n)$ is not independent of $p$. This is in fact obvious as $\lambda_{p}(\infty)=2 p$, which is dependent on $p$, although $\lambda_{p}(0)=2$ is independent of $p$.

\section{Bandwidth}

Extending the model to cases with $p>2$ suggests a natural generalization. The restriction that at most two walkers can meet at any one point - which is the difference between the standard $\infty$-friendly model (also known as the GV $\infty$-friendly model) and the TK $\infty$-friendly model - seems to be relatively arbitrary. This suggests that we can create another parameter of the model, called bandwidth (denoted by $b$ ), which denotes the number of walkers that can meet at any point or line. Of course, this is only relevant for $p>2$. The $\mathrm{GV} \infty$-friendly model then has $b=2$, the smallest possible value it can take without forcing the walkers to be vicious, and the TK model has $b=p$, the largest possible value it can take.

We calculated some generating functions for $p=3, b=3$, for small widths. Because the number of walks grows much more rapidly with $n$ than in the case $p=2$ or even $p=3, b=2$, direct enumeration quickly became infeasible. In order to generate an appropriate number of terms, we used the method of recurrences devised by Gessel ([Ges]). The most notable feature of these generating functions is that the degree of both numerator and denominator grows more rapidly with $n$ than in the $b=2$ case. Also the pattern of 'fixed' coefficients observed above appears to be absent.

Theorem 6.1. The number of 1-friendly walks with $m$ steps, for fixed $p$ and $L$, is independent of $b$ for $b>1$.

Proof. Since there are exactly two ways to reach any point, if we require more than two walkers to reach the same site, then at least 2 walkers must share a bond. However, the walkers are osculating, so this is impossible. Thus the number of walks is the same as the number of walks with $b=2$. 


\section{Acknowledgements}

We would like to thank Christian Krattenthaler for helpful discussions and for directing us to his paper ([Kra01]), and Ira Gessel for describing a method to generate longer series ([Ges]). We would also like to thank the anonymous referee who proved Theorems 4.1 and 4.2 ; in the previous draft they were conjectures.

Yao-ban Chan would like to thank the Australian Government and CSIRO for the scholarships they have provided him with. AJG wishes to acknowledge the support of the Australian Research Council Centre of Excellence in the Mathematics and Statistics of Complex Systems.

\section{References}

[Ges] Ira Gessel. Private communication.

[GOV98] Anthony J. Guttmann, Aleks L. Owczarek, and Xavier G. Viennot. Vicious walkers and Young tableaux I: without walls. J. Phys. A: Math. Gen., 31:8123-8135, 1998.

[GV02] Anthony J. Guttmann and Markus Vöge. Lattice paths: vicious walkers and friendly walkers. J. Stat. Plan. and Inf., 101:107-131, 2002.

[KGV00] Christian Krattenthaler, Anthony J. Guttmann, and Xavier G. Viennot. Vicious walkers, friendly walkers and Young tableaux: II. With a wall. J. Phys. A: Math. Gen., 33:8835-8866, 2000 .

[KGV03] Christian Krattenthaler, Anthony J. Guttmann, and Xavier G. Viennot. Vicious walkers, friendly walkers and Young tableaux. III. Between two walls. J. Stat. Phys., 110(3-6):10691086, 2003.

[Kra01] Christian Krattenthaler. Permutations with restricted patterns and Dyck paths. Adv. Appl. Math., 27(2-3):510-530, 2001.

[Sta97] Richard Stanley. Enumerative Combinatorics. Cambridge University Press, New York, 1997. 